\title{
Contexto de las especificaciones de regularidad superficial en proyectos de rehabilitación costarricense
}

\section{Surface roughness specifications context in costa rican rehabilitation projects}

\author{
Ing. Verónica Solís Salas \\ Ingeniera Civil \\ veronicass91@gmail.com
}

Fecha de recepción: 31 de marzo de 2018 / Fecha de aprobación: 19 de junio de 2018

\section{RESUMEN}

Para garantizar el desempeño del pavimento en proyectos de rehabilitación con sobrecapa asfáltica es necesaria la implementación de especificaciones técnicas, que permitan evaluar la calidad de la construcción. La aplicabilidad de las especificaciones debe ser validada en términos de valores logrados en proyectos previos, en conjunto con referencias de carácter internacional, donde ya hayan entrado en vigencia especificaciones similares y un seguimiento posterior para validar resultados. En el presente proyecto se analiza la aplicabilidad de las especificaciones establecidas en el Manual de especificaciones generales para la construcción de caminos y puentes de Costa Rica y los requisitos del cartel de licitación $N^{o}$.2014LN-000018-0CV00, para nueve proyectos en Costa Rica intervenidos con sobrecapa asfáltica en los últimos dos años. De la investigación se concluye que los rangos establecidos para las especificaciones costarricenses concuerdan con los establecidos en países como Chile, Colombia, España y Estados Unidos. Adicionalmente, los resultados mostraron que, pese a que tales especificaciones no eran vigentes en el cartel de licitación, se lograron cumplimientos aceptables, lo que indica la aplicabilidad de la normativa a la realidad costarricense. Finalmente, como producto de la investigación, se definieron mejoras a las especificaciones actuales que pueden ser verificadas posteriormente en los nuevos proyectos que entraran en ejecución.

PALABRAS CLAVES: IRI, regularidad superficial, sobrecapas, especificaciones

\begin{abstract}
To guarantee pavement performance in rehabilitation projects with asphalt overlays, the implementation of technical specifications that enables the assessment of its construction quality is necessary. The applicability of the specifications must be validated in terms of achieved values from past projects altogether with international references, where similar specifications have gone into effect, followed up with the validation of the results. In the present project, the applicability of the specifications from the Manual of general specifications for road and bridge constructions in Costa Rica is analyzed with the requirements of bid paper $N^{\circ} 2014 L N-$ 000018-0CV00 for nine projects with asphalt overlay interventions in the last two years. From the investigation it can be concluded that the established range of values in the specifications matches the ones in countries like Chile, Colombia, Spain and United States. Additionally, the results showed that, even though said specifications were not valid for the bid paper, acceptable compliance was achieved, which indicates the applicability of the normative in the Costa Rican reality. Finally, as a product of the investigation, improvements for the current specifications were defined, where these can be verified in actual projects.
\end{abstract}

KEY WORDS: IRI, roughness, overlays, specifications 


\section{INTRODUCCIÓN}

El Índice de Regularidad Internacional, IRI, se calcula utilizando una simulación del modelo de cuarto de carro, que circula sobre mediciones del perfil longitudinal de una carretera a una velocidad de simulación de $80 \mathrm{~km} / \mathrm{h}$. El IRI es utilizado en el control, aceptación y recepción de proyectos nuevos y de rehabilitación de carreteras, ya que permite evaluar y garantizar la funcionalidad del pavimento, brindando mayor seguridad y confort a los conductores, así como disminuir costos de operación y costos de mantenimiento.

Para una correcta aplicación del IRI como indicador de la condición funcional del pavimento es necesario validar las normativas existentes, con el fin de garantizar que las obras viales cumplan con condiciones de calidad, confort y seguridad al usuario de acuerdo a valores que pueden ser alcanzados en campo. Generalmente, en proyectos de rehabilitación se establecen porcentajes de mejora para determinar el porcentaje de pago al constructor de la obra. Estos porcentajes de pago, influenciarán de forma directa en los costos de operación y funcionalidad de la carretera, por lo que deben ser determinados con criterios técnicos de las normativas que, a su vez, permitan una adecuada evaluación de la regularidad superficial del pavimento. A continuación, se comentará acerca de las normativas vigentes en Costa Rica, relacionadas con este tema.

\section{Manual de Especificaciones Generales para la Construc- ción de Carreteras, Caminos y Puentes (CR-2010)}

En la sección 401.17 Control de regularidad (IRI) en sobrecapas, reciclajes con sobrecapas o sobrecapas sobre fresados, de mezcla asfáltica en caliente, se establece los requisitos para el pago de trabajos de rehabilitación asociado a la regularidad superficial del pavimento luego de ser intervenido por una sobrecapa asfáltica y los factores de pago según el porcentaje de mejora en el IRI luego de la rehabilitación.

El porcentaje de mejora del IRI se determina al medir la regularidad superficial del pavimento antes y después de la colocación de la sobrecapa en toda la longitud del proyecto. Dicho porcentaje debe de realizarse con un decimal para segmentos de 100 metros, mediante la Ecuación 1:

$\%$ Mejora $=\left[\frac{I R I_{\text {original }}-I R I_{\text {Final }}}{I R I_{\text {Original }}}\right] * 100$

El factor de pago de la obra se determina según el porcentaje de mejora en el IRI de segmento de 100 metros y el tipo de sobrecapa asfáltica, como se muestra en el Cuadro 1.

Cuadro 1. Factor de pago en sobrecapas según porcentaje de mejora del IRI.

\begin{tabular}{|c|c|c|c|}
\hline $\begin{array}{c}\text { Sobrecapa simple } \\
\text { \% de mejora del IRI }\end{array}$ & $\begin{array}{c}\text { \% de pago del valor de la carpeta } \\
\text { según mejora }^{(1)}\end{array}$ & $\begin{array}{c}\text { Multi-Capa } \\
\text { \% de mejora del IRI }\end{array}$ & $\begin{array}{c}\text { \% de pago del valor de la } \\
\text { carpeta según mejora }^{(2)}\end{array}$ \\
\hline$\geq 50$ & 100 & $40<60$ & 100 \\
\hline $35<50$ & 75 & $30<40$ & 50 \\
\hline $25<35$ & 50 & $20<30$ & 25 \\
\hline $15<25$ & 25 & $<20$ & Rechazo $^{(3)}$ \\
\hline
\end{tabular}

(1) Para sobrecapas sencillas sin ningún otro trabajo previo de corrección como fresado, rectificación y/o prenivelación en más del $25 \%$ de la superficie de la calzada existente.

(2) Para sobrecapas sencillas con otras operaciones previas, tales como fresado, rectificación y/o prenivelado, seguido de uno o más sobrecapas, o dos o más sobrecapas colocadas sobre el pavimento sin fresado, rectificado o prenivelado.

(3) Cuando las correcciones no son permitidas o no son efectuadas por el Contratista. 
Las áreas defectuosas pueden ser corregidas según el método de corrección aprobado por el Ingeniero del proyecto. En caso de realizar correcciones, se debe obtener nuevamente el IRI en las áreas corregidas, y con el valor obtenido se sustituye el original.

El cálculo del IRI para sobrecapas asfálticas debe realizarse con equipos clase 1, según la clasificación de equipos definida por el Banco Mundial, y reportarse para secciones de 100 metros. Se asume que el cálculo del IRI es determinado con las indicaciones de la sección 401.16, debido a que no se especifica un método. Se establece que el IRI se debe reportar en tramos de 100 metros y, en caso de presentar singularidades, éstas afectan el sector de 100 metros, por lo cual no se tomará en cuenta en la evaluación. (Ministerio de Obras Públicas y Transportes, 2010)

\section{Cartel de Licitación Pública N. 2014LN-000018-0CV00}

Esta licitación establece en la sección: Control de regularidad (IRI) en sobrecapas, reciclajes con sobrecapas o sobrecapas sobre fresados, de mezcla asfáltica en caliente (Mantenimiento Periódico), que el pago para proyectos de rehabilitación se calculará de igual manera que lo establecido en el CR-2010; sin embargo, se realiza una enmienda que modifica los criterios de aceptación y pago.

Se establece que el uso de estas estrategias de intervención se debe justificar técnica y económicamente por parte del Ingeniero en proyectos que presentan valores de IRI mayores a $6,4 \mathrm{~m} / \mathrm{km}$ junto con una capacidad estructural deficiente. (Consejo Nacional de Vialidad, 2014)

En el cartel se establecen requisitos de aceptación que implicaría el pago del 100\% de las labores realizadas según el valor inicial del IRI. Estos requisitos son presentados en el Cuadro 2.

\section{Cuadro 2. Especificación de IRI recomendada para colocación de sobrecapas asfálticas en proyectos de conservación vial según Enmienda No2 al cartel de licitación pública No.2014LN-000018-0CV00}

\begin{tabular}{|c|c|}
\hline $\begin{array}{c}\text { Rango inicial de IRI } \\
(\mathrm{m} / \mathrm{km})\end{array}$ & $\begin{array}{c}\text { IRI Final } \\
(\mathrm{m} / \mathrm{km})\end{array}$ \\
\hline $2,5-3,6$ & Reducción del $10 \%$ \\
\hline $3,6-6,4$ & $\leq 3,2$ \\
\hline$>6,4$ & Reducción del $50 \% \mathrm{y} \leq 5$ \\
\hline
\end{tabular}

Fuente: Adaptado de Consejo Nacional de Vialidad, 2015

El cartel de licitación para el mantenimiento y rehabilitación de la red vial pavimentada indica que la medición del perfil se debe realizar en intervalos de $25 \mathrm{~mm}$. La enmienda $\mathrm{N}^{\circ} 2$ al cartel de licitación indica que la evaluación del IRI se debe realizar en tramos de 100 metros, al igual que el CR-2010, donde el cálculo se debe realizar antes e inmediatamente después de la intervención. En caso de incumplimiento el contratista debe corregir las áreas defectuosas en tramos de 100 metros, para alcanzar los valores mostrados en el Cuadro 2, en caso de no cumplirlos la obra será rechazada. (Consejo Nacional de Vialidad, 2015)

\section{OBJETIVOS}

\section{Objetivo General}

Evaluar la aplicación de las especificaciones del Índice de Regularidad Internacional (IRI) en los proyectos de rehabilitación carreteros en Costa Rica.

\section{Objetivos Específicos}

- Procesar datos obtenidos en campo por la Unidad de Auditoría Técnica y la Unidad de Gestión y Evaluación de Red Vial Nacional del LanammeUCR-PITRA en proyectos de rehabilitación para la evaluación de la normativa nacional de IRI.

- Analizar la información recopilada y procesada de los proyectos bajo las especificaciones establecidas en la sección 401.17 del CR-2010 y en el cartel de licitación No. 2014LN000018-0CV00.

- Dar recomendaciones para la normativa costarricense utilizada en proyectos de rehabilitación carreteros.

\section{METODOLOGÍA}

Para la realización del proyecto, se recopilaron datos del IRI en pavimentos flexibles de rutas pertenecientes a la Red Vial Nacional Pavimentada. La información de la condición previa a la colocación de la sobrecapa asfáltica se obtuvo de la Evaluación de Red Vial Nacional del 2015, facilitados por la Unidad de Gestión y Evaluación de Red Vial Nacional del Programa de Infraestructura del Transporte (PITRA) del LanammeUCR. Para la condición posterior, se realizaron mediciones de perfil y cálculo del IRI en nueve proyectos nuevos de rehabilitación con sobrecapa asfáltica, facilitados por la Unidad de Auditoría Técnica del PITRA.

Después de recopilar los datos de IRI de cada uno de los proyectos, se procesó la información para su posterior análisis. El procesamiento de información se realizó siguiendo el procedimiento establecido en el CR-2010 y en el cartel de licitación para conservación vial. Se obtuvieron resultados individuales para cada proyecto y se realizaron análisis estadísticos con t-pareada para determinar si las especificaciones fueran adecuadas a la realidad de los proyectos de rehabilitación. 


\section{DESARROLLO}

Para el desarrollo de la investigación se seleccionaron nueve proyectos de la Red Vial Nacional Pavimentada intervenidos mediante la colocación de una sobrecapa asfáltica, los cuales se listan a continuación:
Los datos de IRI para cada uno de los proyectos, antes de la rehabilitación, utilizados en el desarrollo de esta investigación se adquieren de Evaluación de la Red Vial Nacional Pavimentada de Costa Rica del periodo 2014-2015. Estos datos presentan el IRI en intervalos de $100 \mathrm{~m}$, de igual forma como lo solicita la normativa, sin embargo, no descartan las singularidades presentes y son calculados en un solo sentido del proyecto, esto debido a que se utilizan los datos de la Evaluación de la Red Vial Nacional realizada por el LanammeUCR.

Cuadro 3. Rutas y secciones de control intervenidos mediante sobrecapa asfáltica

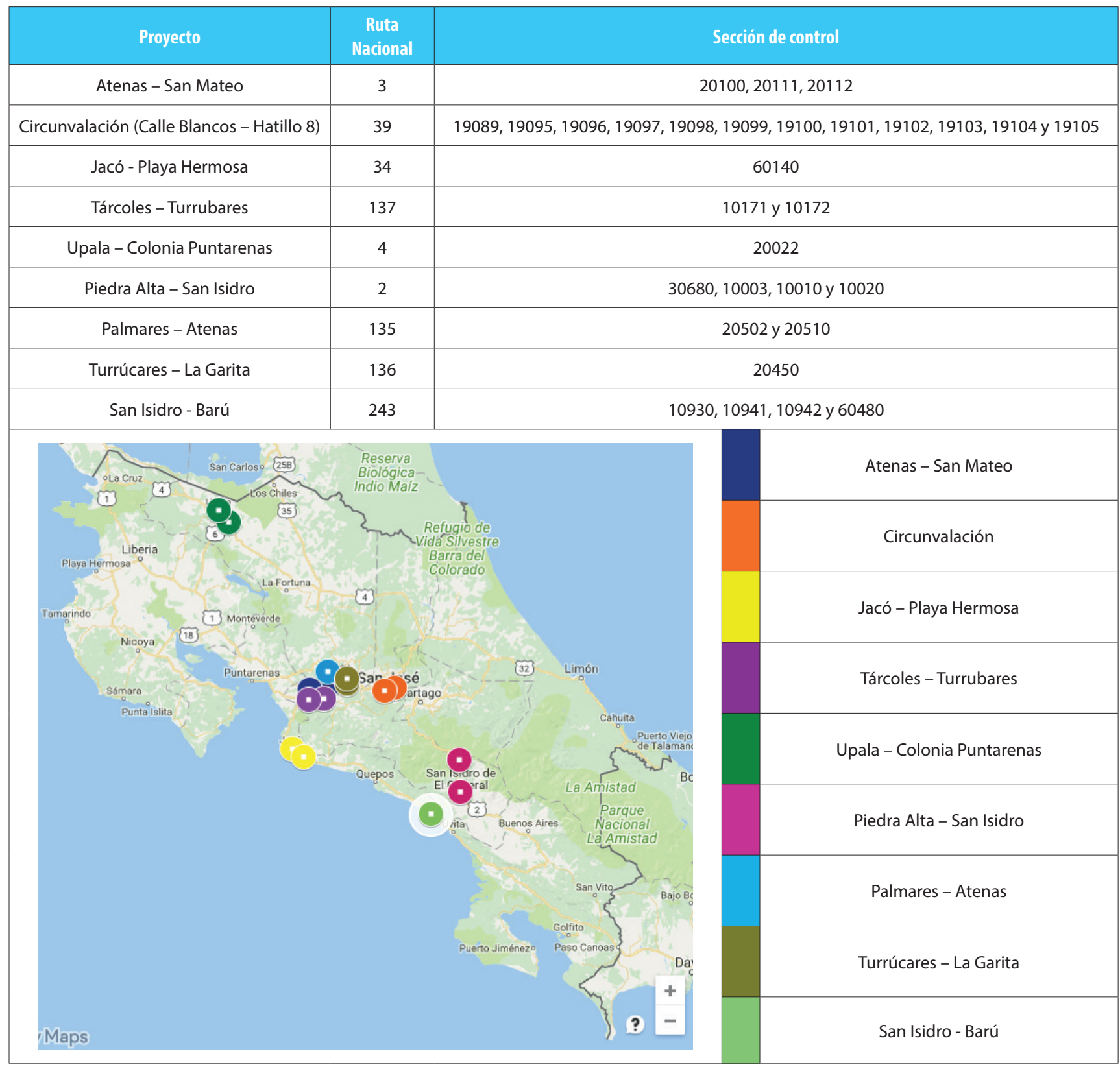


Para el caso de los datos de IRI después de la aplicación de la sobrecapa asfáltica se realizó la medición del perfil longitudinal cada $10 \mathrm{~cm}$, utilizando un perfilómetro inercial, por lo que se promedian diez valores consecutivos para determinar el IRI de $100 \mathrm{~m}$.

En el procesamiento de los datos no se descartan las singularidades presentes en el proyecto, sin embargo, para el análisis probabilístico se descartan los valores atípicos presentes, los cuales podrían representar la presencia de alguna singularidad en el sector analizado o secciones que podrían ser corregidas. Además, debido a que la normativa realiza una comparación antes y después de la colocación de una sobrecapa asfáltica fue necesario el uso del software ArcGIS 10.4.1 para darle un kilometraje a cada sección de 100 m evaluada y lograr realizar una comparación de aproximadamente el mismo sector. Se define como valores atípicos aquellos valores mayores a 1,5 veces el rango intercuartil que podrían representar alguna singularidad.

\section{ANÁLISIS DE RESULTADOS}

Los proyectos de sobrecapa se clasifican en dos tipos: sobrecapa simple de mejora y multicapa de mejora. Se realiza un Análisis de Varianza (ANOVA) para determinar si existe variabilidad entre los proyectos, comparando la condición inicial de los proyectos, basándose en el IRI inicial según el tipo de sobrecapa asfáltica.

El análisis de la especificación se realiza en tramos donde la técnica de intervención aplicada es la correcta según la capacidad estructural y funcional de la vía basado en lo especificado en el informe de Evaluación de la Red Vial Nacional 2015 (LanammeUCR, 2015), porloqueseeliminandelproyectoCalleBlancos-Hatillo 8 los tramos pertenecientes a las secciones de control 19095, 19102, 19103 y 19105. La eliminación de estos tramos se realiza para evaluar si se cumplen los requisitos establecidos cuando la técnica de rehabilitación es la que se propone en la ERVN2015.

\section{CR-2010}

\section{Sobrecapa simple de mejora}

En la Figura 1, se muestra una comparación mediante diagrama de cajas de diversos proyectos clasificados como sobrecapa simple, donde se observa diferencias por proyecto. Los resultados del ANOVA rechazan la hipótesis de igualdad entre las medias de los valores iniciales de IRI de los proyectos clasificados como sobrecapa simple de mejora. Las comparaciones realizadas en parejas mediante Tukey obtienen 5 diferentes agrupaciones para los 6 proyectos de sobrecapa lo que muestra una alta variabilidad entre la condición inicial de cada proyecto, por lo que se realiza un análisis descriptivo al aplicar la normativa establecida en la sección 401.17 del CR-2010.

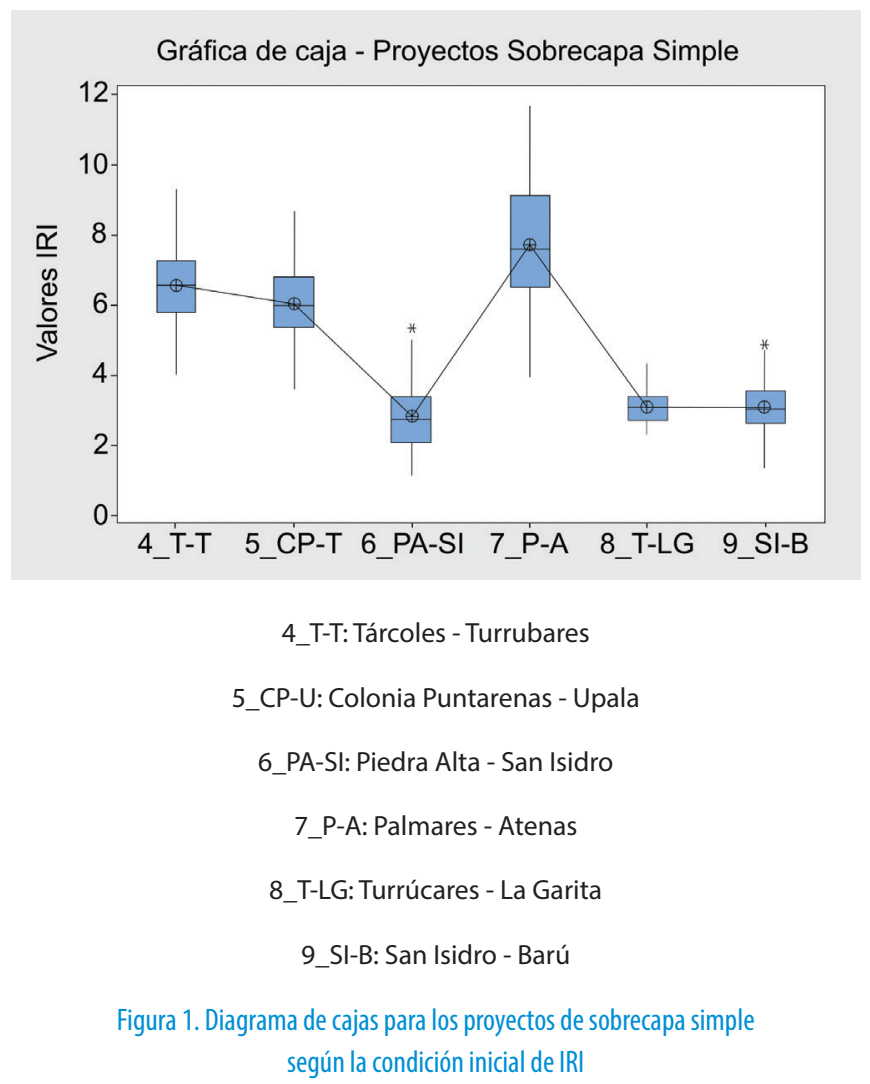

En la Figura 2 y Cuadro 3, se muestra que únicamente un 16\% de los tramos evaluados clasifican en condición de aceptación, por lo que reciben un $100 \%$ del pago por labores realizadas. Por otro lado un $37 \%$ del total de tramos presentan porcentajes de mejora inferiores al 15\%, implicando el rechazo de estos tramos.

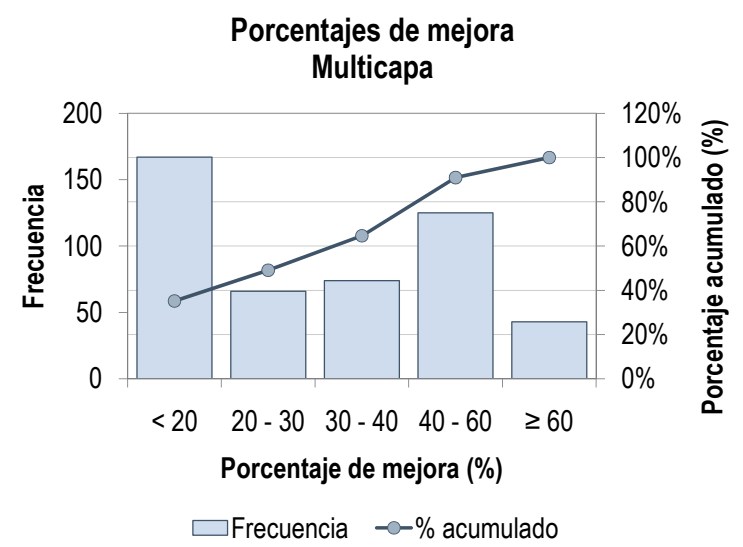

Figura 2. Distribución tramos según porcentajes de mejora para proyectos de sobrecapa simple. 


\begin{tabular}{|l|c|c|c|c|c|}
\hline \multirow{2}{*}{ Proyecto } & \multicolumn{5}{|c|}{ Porcentaje de pago según CR-2010 } \\
\cline { 2 - 6 } & $100 \%$ & $75 \%$ & $50 \%$ & $\mathbf{2 5} \%$ & Rechazo \\
\hline Sobrecapa simple & $16 \%$ & $22 \%$ & $13 \%$ & $13 \%$ & $37 \%$ \\
\hline
\end{tabular}

El valor promedio de IRI inicial para todos los tramos era de $4,0 \mathrm{~m} /$ $\mathrm{km}$ y luego de la intervención fue de $2,7 \mathrm{~m} / \mathrm{km}$, lo que implica una disminución promedio de 1,3 m/km. En todos los tramos de sobrecapa simple se obtuvo un porcentaje de mejora promedio de $22 \%$, lo que implica un pago del $25 \%$ de las obras. Se obtiene que un $21 \%$ de los tramos empeoran la condición de regularidad, mientras que un $79 \%$ presenta alguna mejoría luego de la aplicación de la sobrecapa asfáltica, sin embargo, no se logran cumplir las especificaciones del CR-2010.

\section{Multicapa de mejora}

En la Figura 3, se muestra un diagrama de cajas de los valores iniciales de IRI para cada proyecto clasificado como multicapa de mejora, donde se observa diferencias por proyecto. Se realiza un ANOVA para determinar si los proyectos cuentan con condiciones de regularidad similares previas a su intervención, el cual indica que al menos una de las medias es diferente. Por ser únicamente tres proyectos de multicapa de mejora se realiza un análisis descriptivo, en lugar de estadístico, de la aplicación de normativa del CR-2010. En la Figura 4 se presenta estos resultados y la distribución de los tramos restantes según el porcentaje de mejora.

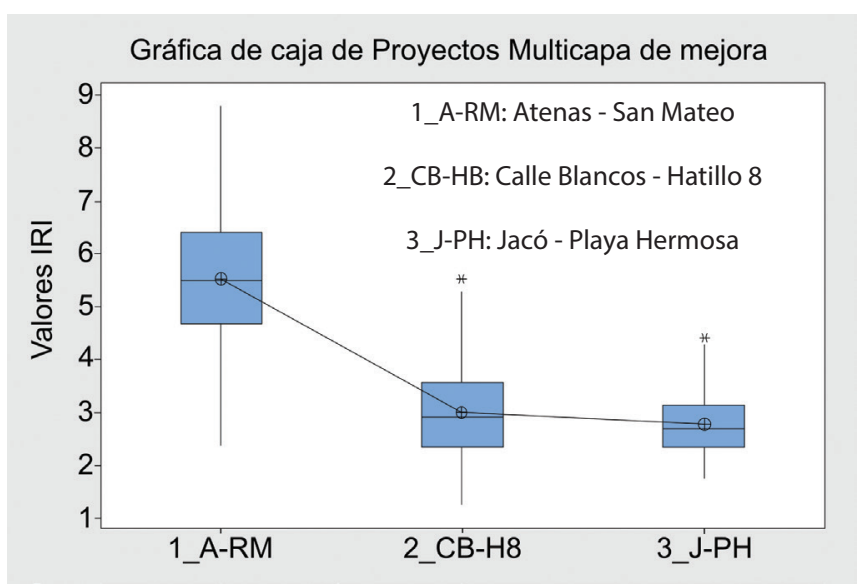

Figura 3. Diagrama de cajas para los proyectos de multicapa de mejora según la condición inicial de IRI

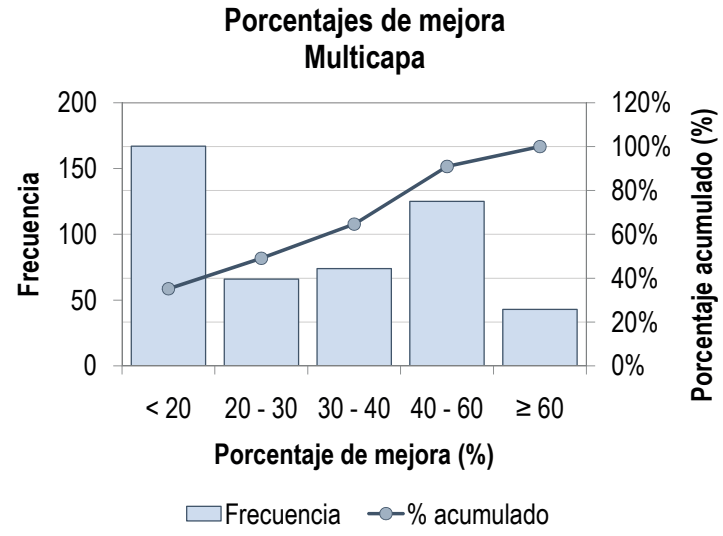

Figura 4. Distribución tramos según porcentajes de mejora para proyectos de multicapa de mejora.

En el Cuadro 5 se puede observar que un 9\% de los tramos cumplen con el requisito para el criterio del pago del 100\%; mientras que un $35 \%$ presentan porcentajes de mejora inferiores al 20\%, lo que implica el rechazo de dichos tramos.

\section{Cuadro 5. Resultados de evaluación según sección 401.17 del} CR-2010, proyectos de multicapa de mejora

\begin{tabular}{|l|c|c|c|c|c|}
\hline \multirow{2}{*}{ Proyecto } & \multicolumn{5}{|c|}{ Porcentaje de pago según CR-2010 } \\
\cline { 2 - 6 } & $\mathbf{1 0 0 \%}$ & $\mathbf{7 5 \%}$ & $\mathbf{5 0 \%}$ & $\mathbf{2 5 \%}$ & Rechazo \\
\hline Multicapa de mejora & $\mathbf{9} \%$ & $\mathbf{2 6 \%}$ & $16 \%$ & $14 \%$ & $35 \%$ \\
\hline
\end{tabular}

Para la condición inicial se presenta un valor promedio de IRI de 4,1 $\mathrm{m} / \mathrm{km}$ y después de la intervención se obtiene un valor de $2,8 \mathrm{~m} / \mathrm{km}$. El porcentaje de mejora promedio fue de $22 \%$, lo que implica un $25 \%$ del pago de obras. Se encuentra un $19 \%$ que empeoran su condición de regularidad superficial, mientras que un $81 \%$ presentan alguna mejoría en la regularidad superficial, sin embargo, solamente el 8\% alcanzan la condición de aceptación de pago del $100 \%$, por lo que no se cumplen los requisitos establecidos en el CR-2010.

\section{Cartel de Licitación}

Debido a las condiciones iniciales variables, se realiza un análisis descriptivo de los tramos de los nueve proyectos mediante los requisitos de aceptación para sobrecapas asfálticas establecidos en la enmienda No2 del cartel de licitación No.2014LN-0000180CV00, según el tipo de tratamiento para compararlo con el análisis realizado para la sección 401.17 del CR-2010. 
El Cuadro 6, presenta los resultados de la evaluación de los proyectos clasificados como sobrecapa simple.

Cuadro 6. Resultados de evaluación del cartel de licitación en proyectos de sobrecapa simple

\begin{tabular}{|c|c|c|c|c|c|c|}
\hline $\begin{array}{l}\text { Rango inicial de } \\
\text { IRI (m/ } / \mathrm{km})\end{array}$ & $\begin{array}{l}\text { Requisito de IRI } \\
\text { Final (m/km) }\end{array}$ & $\begin{array}{c}\text { Cantidad } \\
\text { de } \\
\text { tramos }\end{array}$ & $\begin{array}{c}\text { Tramos } \\
\text { aprobados }\end{array}$ & $\begin{array}{c}\% \\
\text { Aprobados }\end{array}$ & $\begin{array}{l}\text { Tramos } \\
\text { rechazados }\end{array}$ & $\begin{array}{c}\% \\
\text { Rechazados }\end{array}$ \\
\hline$<2,5$ & - & 204 & 204 & $100 \%$ & 0 & $0 \%$ \\
\hline $2,5-3,6$ & $\begin{array}{c}\text { Reducción del } \\
10 \%\end{array}$ & 380 & 238 & $63 \%$ & 142 & $37 \%$ \\
\hline $3,6-6,4$ & $\leq 3,2$ & 293 & 214 & $73 \%$ & 79 & $27 \%$ \\
\hline$>6,4$ & $\begin{array}{c}\text { Reducción del } \\
50 \% y \leq 5\end{array}$ & 143 & 70 & $49 \%$ & 73 & $51 \%$ \\
\hline \multicolumn{2}{|c|}{ Total } & 1020 & 726 & $71 \%$ & 294 & $29 \%$ \\
\hline
\end{tabular}

En la Figura 5, se observa que un 37\% de los tramos tienen valores iniciales de IRI entre 2,5 y $3,6 \mathrm{~m} / \mathrm{km}$, de los cuales un $63 \%$ tienen aprobación. Los tramos en el rango de 3,6 y 6,4 m/ km de IRI inicial, representan un $29 \%$ del total y poseen un porcentaje de aprobación del 73\% según el cartel de licitación. Por su parte, los tramos con un valor inicial de IRI mayor a $6,4 \mathrm{~m} / \mathrm{km}$, el cual representaba el 14\% de los tramos, presentaron un $49 \%$ de aprobación. En general, los resultados obtenidos indican que los proyectos de sobrecapa simple tuvieron un $71 \%$ de aprobación y un $29 \%$ de rechazo.

\section{Multicapa de mejora}

El Cuadro 7, presenta los resultados de evaluar los tramos de los proyectos de multicapa de mejora según los requisitos del cartel de licitación.

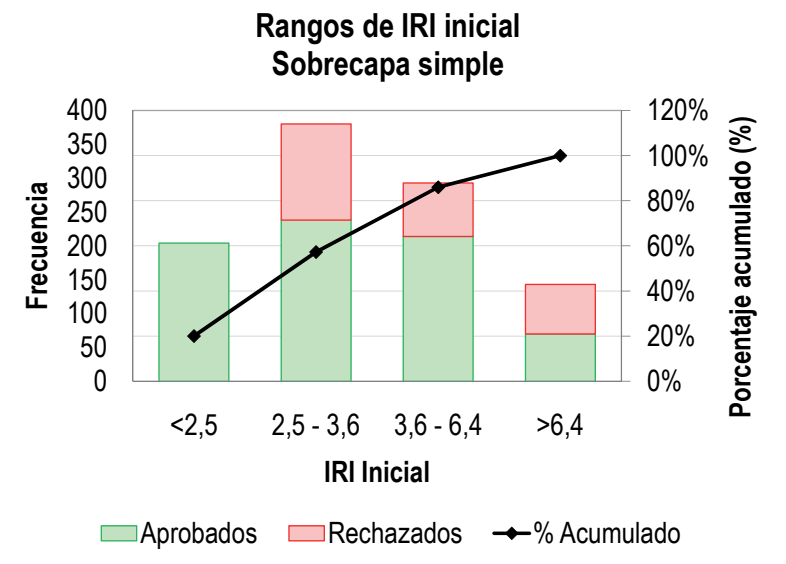

Figura 5. Distribución tramos según valor inicial de IRI, proyectos de sobrecapa simple.

Cuadro 7. Resultados de evaluación del cartel de licitación en proyectos de multicapa de mejora.

\begin{tabular}{|c|c|c|c|c|c|c|}
\hline Rango inicial de IRI (m/km) & $\begin{array}{l}\text { Requisito de IRI Final } \\
(\mathrm{m} / \mathrm{km})\end{array}$ & $\begin{array}{l}\text { Cantidad de } \\
\text { tramos }\end{array}$ & $\begin{array}{l}\text { Tramos } \\
\text { aprobados }\end{array}$ & \% Aprobados & $\begin{array}{l}\text { Tramos } \\
\text { rechazados }\end{array}$ & $\%$ Rechazados \\
\hline$<2,5$ & - & 91 & 91 & $100 \%$ & 0 & $0 \%$ \\
\hline $2,5-3,6$ & Reducción del 10\% & 135 & 85 & $63 \%$ & 50 & $37 \%$ \\
\hline $3,6-6,4$ & $\leq 3,2$ & 196 & 123 & $63 \%$ & 73 & $37 \%$ \\
\hline$>6,4$ & $\begin{array}{l}\text { Reducción del } 50 \% \\
y \leq 5\end{array}$ & 53 & 35 & $66 \%$ & 18 & $34 \%$ \\
\hline \multicolumn{2}{|c|}{ Total } & 475 & 334 & $70 \%$ & 141 & $30 \%$ \\
\hline
\end{tabular}


En la Figura 6, se observa que un $41 \%$ del total corresponden a tramos con un IRI inicial entre 3,6 y 6,4 m/ $\mathrm{km}$, de los cuales $63 \%$ son aprobados; un $28 \%$ del total representan tramos con un valor inicial de IRI entre 2,5 y 3,6 m/ $\mathrm{km}$ con un $63 \%$ de aprobación. Además, un $11 \%$ del total poseen tramos con valores de IRI mayores a $6,4 \mathrm{~m} / \mathrm{km}$, de los cuales $66 \%$ son aprobados. Los proyectos de multicapa de mejora tienen un 70\% que cumplen con los requisitos de aprobación establecidos por el cartel de licitación y un 30\% son rechazados.

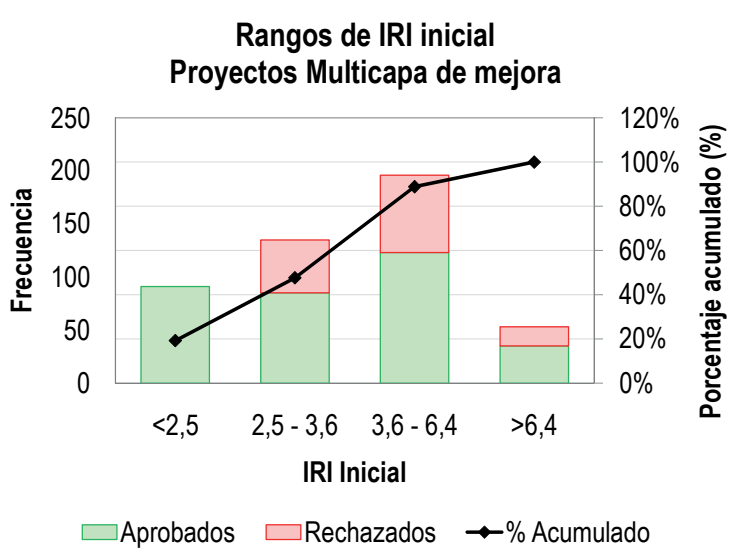

Figura 6. Distribución tramos según valor inicial de IRI, proyectos de multicapa de mejora

\section{CONCLUSIONES}

El IRI es un parámetro ampliamente utilizados en normativas internacionales como parámetro de aceptación tanto de obra nueva como de rehabilitación, pero poco utilizado como criterio para la determinación del factor de pago.

En la normativa costarricense al igual que en la internacional, la determinación del IRI se realiza en tramos de $100 \mathrm{~m}$.

En ciertos proyectos, se realizó una intervención más rigurosa de la que se necesitaba según la condición del pavimento, evidenciando un uso no eficiente de los recursos y no apropiada gestión de proyectos.

En la Figura 7 se muestra la condición antes y después de la intervención. Los resultados obtenidos mostraron que, en ocho de los nueve proyectos analizados, se presentó disminución en el valor de IRI, indicando que una mejor condición de regularidad al rehabilitar los proyectos mediante colocación de una sobrecapa asfáltica.
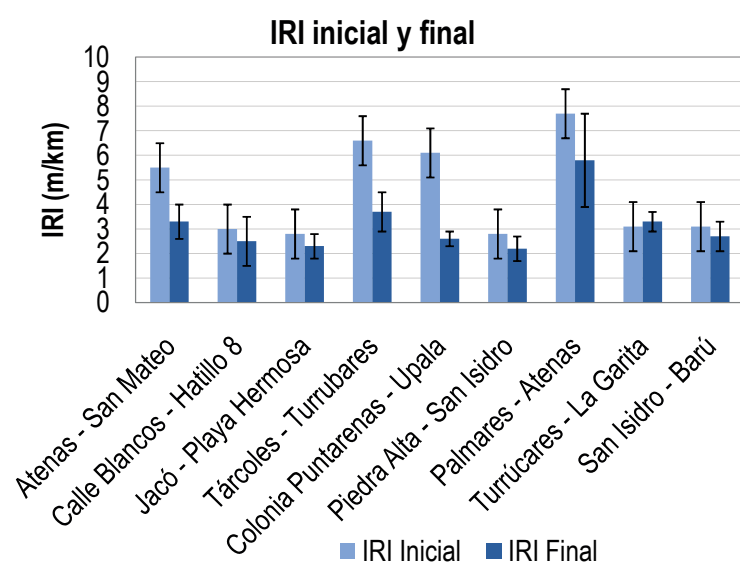

Figura 7. Esquema del análisis de datos

\section{CR-2010}

La evaluación de proyectos mediante esta normativa, utilizando los porcentajes de mejora establecidos para la aceptación y control de pago, no fue adecuada para la realidad nacional debido a factores como condición inicial de proyectos, gestión de proyectos y experiencia en procesos constructivos para mejorar regularidad superficial. En el Cuadro 8 se presenta el resumen de los resultados obtenidos según el factor de pago para cada proyecto analizado anteriormente.

\section{Cuadro 8. Resultados obtenidos de la evaluación mediante la sección 401.17 del CR-2010 para todos los proyectos}

\begin{tabular}{|c|c|c|c|c|c|}
\hline \multirow{2}{*}{ Proyecto } & \multicolumn{4}{|c|}{ Porcentajes de pago según CR-2010 } \\
\cline { 2 - 6 } & $\mathbf{1 0 0} \%$ & $\mathbf{7 5 \%}$ & $\mathbf{5 0} \%$ & $\mathbf{2 5} \%$ & Rechazo \\
\hline Atenas - San Mateo & $13 \%$ & $36 \%$ & $19 \%$ & $16 \%$ & $17 \%$ \\
\hline $\begin{array}{c}\text { Circunvalación (Calle } \\
\text { Blancos - Hatillo 8) }\end{array}$ & $4 \%$ & $24 \%$ & $11 \%$ & $14 \%$ & $47 \%$ \\
\hline Jacó - Playa Hermosa & $2 \%$ & $12 \%$ & $19 \%$ & $15 \%$ & $52 \%$ \\
\hline $\begin{array}{c}\text { Tárcoles - Turrubares } \\
\text { Upala - Colonia } \\
\text { Puntarenas }\end{array}$ & $28 \%$ & $59 \%$ & $9 \%$ & $3 \%$ & $1 \%$ \\
\hline $\begin{array}{c}\text { Piedra Alta - } \\
\text { San Isidro }\end{array}$ & $6 \%$ & $16 \%$ & $18 \%$ & $19 \%$ & $41 \%$ \\
\hline Palmares - Atenas & $11 \%$ & $26 \%$ & $15 \%$ & $9 \%$ & $39 \%$ \\
\hline Turrúcares - La Garita & $0 \%$ & $0 \%$ & $0 \%$ & $0 \%$ & $100 \%$ \\
\hline San Isidro - Barú & $0,3 \%$ & $12 \%$ & $16 \%$ & $18 \%$ & $54 \%$ \\
\hline Sobrecapa simple & $16 \%$ & $22 \%$ & $13 \%$ & $13 \%$ & $37 \%$ \\
\hline Multicapa de mejora & $9 \%$ & $26 \%$ & $16 \%$ & $14 \%$ & $35 \%$ \\
\hline
\end{tabular}




\section{Cartel de licitación}

En el Cuadro 9 se muestra un resumen de los resultados obtenidos según la evaluación de estas especificaciones. Según estos resultados, esta especificación es apta para la implementación como normativa de control y aceptación de proyectos de rehabilitación costarricenses, debido a que se adapta a las condiciones presentes en los proyectos. Con esta normativa se obtienen mejores resultados de aceptación, pues considera el valor de IRI inicial del proyecto, parámetro que influye en la condición de regularidad superficial obtenida después de la intervención.

Existe una relación directa entre el valor IRI inicial y la condición final obtenida luego de la intervención, a menor valor inicial de IRI, menor porcentaje de mejora.

No es adecuado diferenciar el tipo de sobrecapa asfáltica como lo especifica el CR-2010, pues no se encontró relación entre la clasificación de sobrecapa simple de mejora y multicapa de mejora, con los porcentajes de mejora obtenidos y cantidad de tramos aprobados según el tipo de intervención.

Aproximadamente el 20\% del total de tramos analizados empeoraron la condición de regularidad superficial, por lo que es necesario implementar las especificaciones para el control de la regularidad superficial, con el objetivo de mejorar los procedimientos constructivos y así obtener estructuras de mejor calidad, disminuir costos de operación vehicular y de mantenimiento de la red vial y alargar la vida útil de los pavimentos.

Se considera el cartel de licitación apto para la implementación del control de proyectos de rehabilitación mediante la colocación de sobrecapa asfáltica.

\section{RECOMENDACIONES}

Se recomienda que, para la comparación entre la condición inicial y final del pavimento rehabilitado, las mediciones estén referenciadas a un punto común. El caso ideal es establecer puntos específicos de referencia para toda la Red Vial Nacional.

Es necesario implementar un programa de gestión de pavimentos que permita aplicar la intervención adecuada según la condición del pavimento, con el fin de aplicar correctamente la normativa y realizar una mejor gestión de los recursos.

Se recomienda indicar que para valores de IRI menores a 2,5 m/km no se permite que la condición de regularidad superficial empeore.

La especificación para control y aceptación según la condición de regularidad superficial debe considerar la condición inicial del pavimento, pues se determinó que este parámetro influye directamente en el valor del IRI después de la rehabilitación. Adicionalmente, los criterios de evaluación de la red, la conservación vial y la recepción de obra nueva deben ser consistentes entre sí.

La especificación para control de regularidad superficial en proyectos de rehabilitación debe tomar en cuenta el tipo de carretera que se va a intervenir, tránsito que recibe y condición inicial del pavimento, de allí la importancia de la generación de esta información para futuros proyectos. Por otra parte, se recomienda el uso de herramientas estadísticas para velar el cumplimiento de la especificación y controlar la variabilidad del proceso constructivo.

Cuadro 9. Resultados obtenidos de la evaluación mediante el cartel de licitación para todos los proyectos.

\begin{tabular}{|c|c|c|c|c|}
\hline \multirow[b]{2}{*}{ Proyecto } & \multicolumn{4}{|c|}{ Porcentaje de tramos aprobados } \\
\hline & $2,5<\mid \mathrm{RI}_{\text {inicial }}<3,6$ & $3,6<\mid \mathrm{RI}_{\text {inicial }}<6,4$ & $6,4<\mathrm{IRI}_{\text {inicial }}$ & $\begin{array}{c}\text { Total de } \\
\text { tramos } \\
\text { aprobados }\end{array}$ \\
\hline Atenas - San Mateo & $29 \%$ & $53 \%$ & $64 \%$ & $54 \%$ \\
\hline Circunvalación (Calle Blancos - Hatillo 8) & $64 \%$ & $80 \%$ & N.A. & $80 \%$ \\
\hline Jacó - Playa Hermosa & $77 \%$ & $93 \%$ & N.A. & $87 \%$ \\
\hline Tárcoles - Turrubares & N.A. & $59 \%$ & $28 \%$ & $42 \%$ \\
\hline Upala - Colonia Puntarenas & $100 \%$ & $97 \%$ & $100 \%$ & $98 \%$ \\
\hline Piedra Alta - San Isidro & $80 \%$ & $90 \%$ & N.A. & $90 \%$ \\
\hline Palmares - Atenas & N.A. & $0 \%$ & $13 \%$ & $9 \%$ \\
\hline Turrúcares - La Garita & $4 \%$ & $0 \%$ & N.A. & $12 \%$ \\
\hline San Isidro - Barú & $57 \%$ & $70 \%$ & N.A. & $67 \%$ \\
\hline Sobrecapa simple & $63 \%$ & $73 \%$ & $49 \%$ & $71 \%$ \\
\hline Multicapa de mejora & $63 \%$ & $63 \%$ & $66 \%$ & $70 \%$ \\
\hline Todos los proyectos & $63 \%$ & $70 \%$ & $54 \%$ & $71 \%$ \\
\hline
\end{tabular}


Finalmente, y con base en los análisis desarrollados se recomienda la modificar los valores mostrados en el Cuadro 2 por los valores mostrados en el Cuadro 10, eliminando la especificación con valores iniciales de IRI de 2,5 a 3,6 m/ km, pues no alcanza una mejora en la clasificación establecida. No obstante, es necesario que ningún tramo empeore la condición de IRI inicial mediante la colocación de una sobrecapa asfáltica.
Cuadro 10. Recomendación para la especificación de IRI para colocación de sobrecapas asfálticas en proyectos de conservación vial.

\begin{tabular}{|c|c|}
\hline Rango inicial de IRI $(\mathrm{m} / \mathrm{km})$ & IRI Final $(\mathrm{m} / \mathrm{km})$ \\
\hline $\mid \mathrm{RI}_{\text {inicial }} \leq 3,6$ & $\leq|\mathrm{R}|_{\text {inicial }} \mathrm{y} \leq 3,2$ \\
\hline $3,6<\mid \mathrm{RI}_{\text {inicial }} \leq 6,4$ & $\leq 3,2$ \\
\hline $\mid \mathrm{RI}_{\text {inicial }}>6,4$ & Reducción del $50 \% \mathrm{y} \leq 5$ \\
\hline
\end{tabular}

\section{REFERENCIAS}

1. Consejo Nacional de Vialidad. (2014). Licitación Pública No.2014LN-000018-0CV00. MP y R: Mantenimiento periódico y rehabilitación del Pavimento de la Red Vial Nacional Pavimentada. San José: Ministerio de Obras Públicas y Transportes.

2. Consejo Nacional de Vialidad. (2015). Licitación Pública No.2014LN-000018-0CV00. Enmienda No.2 al Cartel de Licitación MP y R: Mantenimiento periódico y rehabilitación del Pavimento de la Red Vial Nacional Pavimentada. San José: Ministerio de Obras Públicas y Transportes.

3. Ministerio de Obras Públicas y Transportes. (2010). Manual de Especificaciones Generales para la construcción de carreteras caminos y puentes CR-2010. San José, Costa Rica: Ministerio de Obras Públicas y Transportes.

4. Solís, M. V., Leiva, P., Hidalgo, A. E., \& Loría, L. G. (2017). Contexto del índice de regularidad internacional (IRI) en proyectos de rehabilitación carreteros. San José: Universidad de Costa Rica.

5. LanammeUCR. (2015). Informe de Evaluación de la Red Vial Nacional Pavimentada de Costa Rica, Años 2014-2014 (Primera Edición ed.). Montes de Oca, San José, Costa Rica: Universidad de Costa Rica. 